\title{
Novos registros do gênero Trachelomonas Ehr. (Euglenophyceae) no Parque Estadual Delta do Jacuí e no Rio Grande do Sul, Brasil
}

\author{
Sandra Maria Alves-da-Silva ${ }^{1,3}$ e Adriana Schüler-da-Silva ${ }^{2}$
}

Recebido em 30/08/2004. Aceito em 27/10/2006

\begin{abstract}
RESUMO - (Novos registros do gênero Trachelomonas Ehr. (Euglenophyceae) no Parque Estadual Delta do Jacuí e no Estado do Rio Grande do Sul, Brasil). O levantamento do gênero Trachelomonas Ehr. (Euglenophyceae) em 26 corpos d'água da área do Parque Estadual Delta do Jacuí, localizado a $29^{\circ} 56^{\prime}$ e $30^{\circ} 03^{\prime} \mathrm{S}, 51^{\circ} 12^{\prime}$ e e $51^{\circ} 25^{\prime} \mathrm{W}$, resultou no registro de nove novas citações de espécies de Trachelomonas Ehr. para o Estado do Rio Grande do Sul, são elas: Trachelomonas abrupta Swir. emend. Defl. var. obesa (Playf.) Defl. T. conica Playf., Trachelomonas duquei Conf. \& Nudelman, Trachelomonas megalacantha Cunha var. crenulatocollis Bour. \& Manguin, Trachelomonas verrucosa Stockes var. granulosa (Playf.) Hub.-Pest., Trachelomonas zingeri Roll, três são ainda novos registros para o país: Trachelomonas cylindraceae f. cylindraceae (Playf.) Pop., Trachelomonas helvetica Lemm. emend. Defl. e Trachelomonas splendidissima Middelhoek. São apresentadas descrições, ilustrações, comentários, distribuição geográfica e a amplitude de condições ambientais em que cada táxon foi encontrado.
\end{abstract}

Palavras-chave: Trachelomonas, Euglenophyceae, novas citações, sul do Brasil

\begin{abstract}
New records for the genus Trachelomonas Ehr. (Euglenophyceae) in Jacuí Delta State Park, Rio Grande do Sul, Brazil). A study of the genus Trachelomonas Ehr. (Euglenophyceae) in 26 water bodies at Jacuí Delta State Park near the state capital of Rio Grande do Sul $\left(29^{\circ} 56^{\prime}, 30^{\circ} 03^{\prime} \mathrm{S} ; 5^{\circ} 12^{\prime}, 5^{\circ} 18^{\prime} \mathrm{W}\right)$ revealed nine new records of Trachelomonas species for this state, as follows: Trachelomonas abrupta Swir. emend. Defl. var. obesa (Playf.) Defl., T. conica Playf., Trachelomonas duquei Conf. \& Nudelman, Trachelomonas megalacantha Cunha var. crenulatocollis Bour. \& Manguin, Trachelomonas verrucosa Stockes var. granulosa (Playf.) Hub.-Pest., Trachelomonas zingeri Roll; three are new records for Brazil: Trachelomonas cylindraceae f. cylindraceae (Playf.) Pop., Trachelomonas helvetica Lemm. emend. Defl. and Trachelomonas splendidissima Middelhoek. Descriptions, illustrations, comments, geographic distribution and the range of environmental conditions in which each taxon was found are presented.
\end{abstract}

Key words: Trachelomonas, Euglenophyceae, new records, southern Brazil

\section{Introdução}

$\mathrm{Na}$ divisão Euglenophyta o gênero Trachelomonas Ehr. é o que possui o maior número de representantes. O Rio Grande do Sul caracterizase por apresentar diferentes ambientes aquáticos com elevados teores de matéria orgânica, fator amplamente citado em literatura como capaz de propiciar a ocorrência desta divisão e gênero. Alves-da-Silva \& Hahn (2001) ao realizarem uma revisão de trabalhos envolvendo as Euglenophyta no Rio Grande do Sul até 2000 fazem o registro de 88 táxons de Trachelomonas já identificados no Estado.

O estudo das Euglenophyta integrou um projeto mais amplo dentro do Programa para o Desenvolvimento Racional, Recuperação e Gerenciamento
Ambiental da Bacia Hidrográfica do Guaíba (PróGuaíba) desenvolvido por uma equipe de pesquisadores do Museu Natural da Fundação Zoobotânica do Rio Grande do Sul. Foi desenvolvido no Parque Estadual Delta do Jacuí abrangendo ambientes lênticos e lóticos e permitiu a identificação de mais de 140 representantes desta divisão. Parte do estudo já resultou na publicação de cinco trabalhos (Alves-da-Silva \& Ávila 1997; Alves-da-Silva \& Bridi 2004 a; b; Alvesda-Silva \& Crossetti 1999; Alves da Silva \& Hahn 2004). O presente trabalho é o sexto desta série de estudos da ficoflora de Euglenophyceae no Parque e tem como objetivo apresentar novos registros do gênero Trachelomonas para o Estado do Rio Grande do Sul e Brasil ampliando o conhecimento taxonômico e a distribuição geográfica deste gênero, além de fornecer

\footnotetext{
1 Museu de Ciências Naturais da Fundação Zoobotânica do Rio Grande do Sul, Rua Dr. Salvador França 1427, C. Postal 1188, 90690-000 Porto Alegre, RS, Brasil

2 (adrischuler@yahoo.com.br) completar endereçamento

3 Autor para correspondência: alvesdasilva@fzb.rs.gov.br; salvesilva5@yahoo.com.br
} 
informações de variáveis abióticas dos locais de ocorrência de cada táxon na área de estudo.

\section{Material e métodos}

O Parque Estadual Delta do Jacuí está localizado na região norte do lago Guaíba, entre as coordenadas $29^{\circ} 56^{\prime}$ e $30^{\circ} 03^{\prime} \mathrm{S}, 51^{\circ} 12^{\prime}$ e $51^{\circ} 25^{\prime} \mathrm{W}$ (Fig. 1 ), uma das mais expressivas áreas naturais da região metropolitana de Porto Alegre, abrangendo uma área de 17.245 ha formado por uma grande variedade de ambientes aquáticos apresentando uma alta diversidade de espécies da fauna e flora. O clima local segundo classificação de Köppen é do tipo Cfa ou subtropical úmido. Neste parque foram analisadas amostras de rede coletadas em 1993/1994 e 1998/1999, em oito margens de ilhas, nove sacos, quatro desembocaduras de rios, duas foz de arroios e três canais.

Os novos registros de Trachelomonas para o Estado ocorreram em 19 amostras, sendo 18 de rede e uma de espremido de macrófitas aquáticas e encontram-se depositadas no Herbário Prof. Dr. Alarich R.H. Schultz (HAS) do Museu de Ciências Naturais da Fundação Zoobotânica do Rio Grande do Sul, sob os números HAS26327, HAS26332, HAS26333, HAS27079, HAS34632, HAS34635, HAS34791, HAS34801, HAS34825, HAS34881, HAS34885, HAS34908, HAS34922, HAS34945, HAS34947, HAS34959, HAS34992, HAS34994, HAS34998.

Além da coleta do material biológico, foram realizadas coletas de água para determinação das seguintes variáveis: amônio, nitrato, oxigênio dissolvido, demanda bioquímica de oxigênio, ortofosfato, matéria orgânica e coliformes fecais segundo APHA (1992). Em campo foram medidos o $\mathrm{pH}$, condutividade elétrica, transparência e temperatura da água, nas amostragens de 1998/1999, exceto nas amostragens de 1993/1994 em que foram medidos apenas o $\mathrm{pH}$ e a temperatura da água (Tab. 2).

As amostras foram coletadas com rede de plâncton com malha de $25 \mu \mathrm{m}$ de abertura e subdivididas em duas partes: uma parte foi fixada em campo, com solução de "Transeau" (nas amostras 1993/1994) ou com formol 4\% (nas amostras 1998/1999), e parte foi mantida viva para observação de características morfológicas indispensáveis à identificação taxonômica dos gêneros.

Para análise do material utilizou-se microscópio óptico Leitz, modelo Dialux, sendo executados desenhos com auxílio de câmara clara e efetuadas medições com ocular micrometrada.

Foram considerados táxons raros aqueles que foram encontrados em um a no máximo três locais onde ocorreram novos registros de Trachelomonas.

Para identificação em nível específico e infraespecífico foram utilizadas obras básicas como: Balech (1944), Deflandre (1926), Conrad \& Van Meel (1952), Huber-Pestalozzi (1955), Németh (1980), Starmach (1983), Tell \& Conforti (1986), Wolowski (1998), além de trabalhos recentes.

\section{Resultados e discussão}

A distribuição dos representantes de Trachelomonas no Parque são apresentadas na Tab. 1, e as variáveis ambientais em que cada táxon ocorreu durante o período de estudo constam da Tab. 2.

Foram analisadas 85 amostras, 80 com representantes de Trachelomonas, mas em somente 19 amostras foram registradas novas citações de ocorrência do gênero Trachelomonas para o Parque Estadual Delta do Jacuí e Estado do Rio Grande do
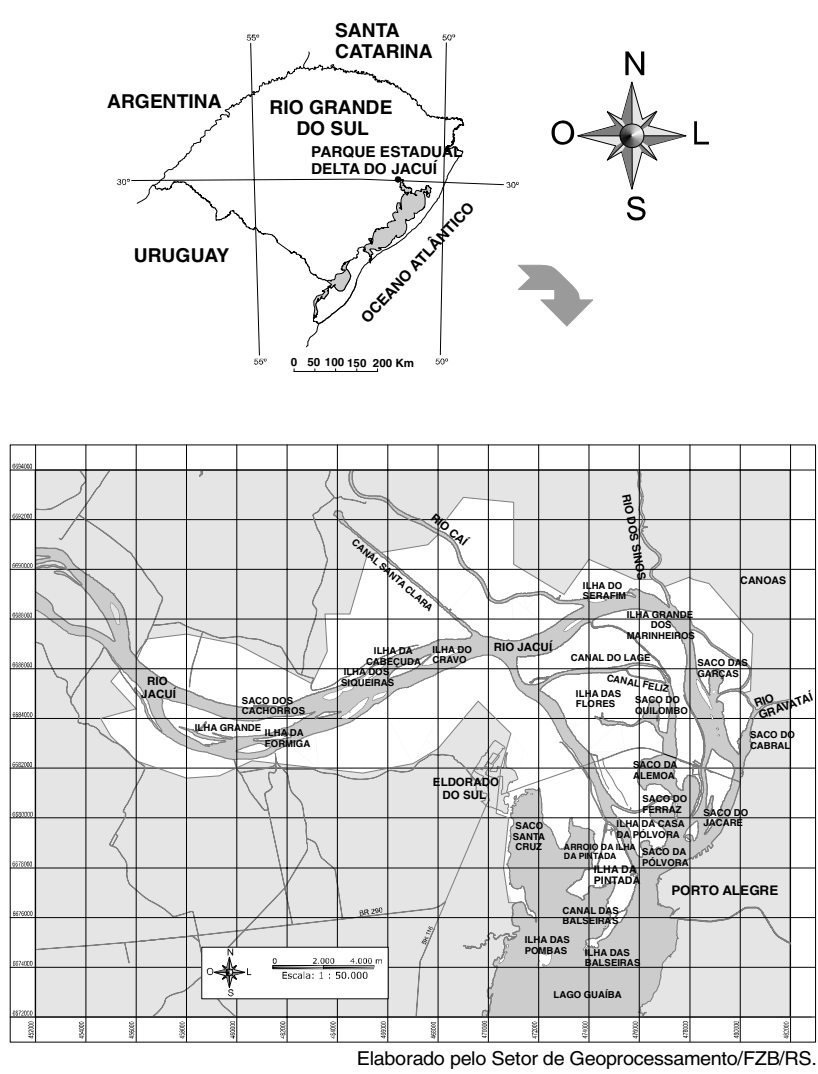

Figura 1. Mapa da área do Parque Estadual Delta do Jacuí, Rio Grande do Sul, Brasil, com os locais de coleta. 
Tabela 1. Distribuição dos novos registros de Trachelomonas Ehr. em biótopos aquáticos no Parque Estadual Delta do Jacuí com seus números de registro no Herbário Prof. Dr. Alarich R.H. Schultz (HAS).

\begin{tabular}{|c|c|c|c|c|c|c|c|}
\hline $\mathrm{N}^{\mathrm{o}} \mathrm{HAS}$ & $\begin{array}{l}\text { Ilha do } \\
\text { Cravo } \\
26327\end{array}$ & $\begin{array}{l}\text { Foz Sanga } \\
\text { das Pedras } \\
26332\end{array}$ & $\begin{array}{l}\text { Ilha do } \\
\text { Siqueira } \\
26333\end{array}$ & $\begin{array}{l}\text { Saco do } \\
\text { Jacaré } \\
27079\end{array}$ & $\begin{array}{l}\text { Saco do } \\
\text { Cabral } \\
34632\end{array}$ & $\begin{array}{l}\text { Ilha do } \\
\text { Serafim } \\
34635\end{array}$ & $\begin{array}{c}\text { Saco do } \\
\text { Cabral } \\
34791\end{array}$ \\
\hline $\begin{array}{l}\text { T. abrupta var. obesa } \\
\text { T. conica } \\
\text { T. cylindraceae f. cylindraceae } \\
\text { T. duquei } \\
\text { T. helvetica } \\
\text { T. megalacantha var. crenulatocollis } \\
\text { T. splendissima } \\
\text { T. verrucosa var. granulosa } \\
\text { T. zingeri }\end{array}$ & $X$ & $\begin{array}{l}X \\
X \\
X\end{array}$ & $X$ & $\mathrm{X}$ & $X$ & & $X$ \\
\hline $\begin{array}{l}\text { Espécies/Locais de coleta/ } \\
\qquad N^{o} \mathrm{HAS}\end{array}$ & $\begin{array}{l}\text { Saco do } \\
\text { Jacaré } \\
34801\end{array}$ & $\begin{array}{l}\text { Foz Rio } \\
\text { Caí } \\
34825\end{array}$ & $\begin{array}{l}\text { Ilha } \\
\text { SantaCruz } \\
34881\end{array}$ & $\begin{array}{l}\text { Ilha do } \\
\text { Serafim } \\
34885\end{array}$ & $\begin{array}{c}\text { Canal das } \\
\text { Balseiras } \\
34908\end{array}$ & $\begin{array}{c}\text { Saco } \\
\text { Santa Cruz } \\
34922\end{array}$ & $\begin{array}{c}\text { Saco do } \\
\text { Cabral } \\
34945\end{array}$ \\
\hline $\begin{array}{l}\text { T. abrupta var. obesa } \\
\text { T. conica } \\
\text { T. cylindraceae f. cylindraceae } \\
\text { T. duquei } \\
\text { T. helvetica } \\
\text { T. megalacantha var. crenulatocollis } \\
\text { T. splendissima } \\
\text { T. verrucosa var. granulosa } \\
\text { T. zingeri }\end{array}$ & $X$ & & $\mathrm{X}$ & $X$ & $X$ & $X$ & $X$ \\
\hline $\begin{array}{l}\text { Espécies/Locais de coleta/ } \\
\qquad N^{\circ} \text { HAS }\end{array}$ & $\begin{array}{l}\text { Saco do } \\
\text { Cabral } \\
34947\end{array}$ & $\begin{array}{l}\text { Saco do } \\
\text { Cabral } \\
34959\end{array}$ & $\begin{array}{c}\text { Saco dos } \\
\text { Cachorros } \\
34992\end{array}$ & $\begin{array}{c}\text { Ilha do } \\
\text { Siqueira } \\
34994\end{array}$ & $\begin{array}{c}\text { Ilha do } \\
\text { Cravo } \\
34998\end{array}$ & & \\
\hline $\begin{array}{l}\text { T. abrupta var. obesa } \\
\text { T. conica } \\
\text { T. cylindraceae f. cylindraceae } \\
\text { T. duquei } \\
\text { T. helvetica } \\
\text { T. megalacantha var. crenulatocollis } \\
\text { T. splendissima } \\
\text { T. verrucosa var. granulosa } \\
\text { T. zingeri }\end{array}$ & $X$ & $X$ & $X$ & & $X$ & & \\
\hline
\end{tabular}

Sul, totalizando nove táxons específicos e infraespecíficos.

\section{Gênero Trachelomonas Ehr.}

1. Trachelomonas abrupta Swir. emend. Defl. var. obesa (Playf.) Defl. Nemours, p. 93. fig. 353, 362, 363. 1926.

Fig. 2

Lórica amplamente elíptica, 26-27 $\mu \mathrm{m}$ compr.; 21-22,6 larg.; Rc/1 = ca. de 1,2; pólo anterior sem colarinho, poro ca. 3,7 $\mu \mathrm{m}$ diâm.; parede densamente pontuada, escrobiculada, castanho-clara; cloroplastídeos numerosos, ca. $2 \mu \mathrm{m}$ diâm.; pirenóides internos até 4,5 $\mu \mathrm{m}$ diâm.

Material examinado: BRASIL. Rio Grande do Sul: Ilha do Cravo, 15/XII/1993, Costa (HAS26327).

Distribuição geográfica: América do Sul, Europa e Oceania. Primeira citação da variedade para o Estado e País.

Comentários: difere da variedade típica pela forma da lórica e pólos convexos, raramente paralelos. 
Tabela 2. Variáveis ambientais e táxons de Trachelomonas Ehr. no Parque Estadual Delta do Jacuí em abril e dezembro/1993 e de maio/1998 a dezembro/1999. NM = não medido.

\begin{tabular}{|c|c|c|c|c|c|c|c|}
\hline Espécies/Variáveis ambientais & $\begin{array}{c}\text { Amônia } \\
(\mu g \mathrm{~L})\end{array}$ & $\begin{array}{c}\text { Nitrito } \\
(\mu g \mathrm{~L})\end{array}$ & $\begin{array}{c}\text { Nitrato } \\
(\mu g \mathrm{~L})\end{array}$ & $\begin{array}{c}\mathrm{OD} \\
(\mathrm{mg} \mathrm{L})\end{array}$ & $\begin{array}{l}\text { DOB5 } \\
(\mathrm{mg} \mathrm{L})\end{array}$ & $\begin{array}{l}\text { Ortofosfato } \\
\quad(\mu g \mathrm{~L})\end{array}$ & $\begin{array}{l}\text { Matéria } \\
\text { orgânica }\end{array}$ \\
\hline T. abrupta var. obesa & $\mathrm{NM}$ & $\mathrm{NM}$ & NM & $\mathrm{NM}$ & $\mathrm{NM}$ & NM & $\mathrm{NM}$ \\
\hline T. conica & 720 & 50 & 700 & 8 & 1,2 & 360 & 5,1 \\
\hline T. cylindraceae f. cylindraceae & NM & NM & NM & NM & NM & NM & NM \\
\hline T. duquei & $12-600$ & $1-131$ & $500-1300$ & $3,1-6,9$ & $0,1-2,4$ & $90-530$ & $3,3-7,2$ \\
\hline T. helvetica & NM & $\mathrm{NM}$ & NM & NM & NM & NM & NM \\
\hline T. megalacantha var. crenulatocollis & $60-360$ & $7-131$ & $1300-2100$ & $4,4-6,5$ & $1-2,4$ & $340-530$ & $3,4-3,7$ \\
\hline T. splendissima & 330 & 12 & 1500 & 5,2 & 1,6 & 330 & 7 \\
\hline T. verrucosa var. granulosa & NM & NM & NM & NM & NM & NM & NM \\
\hline T. zingeri & $390-600$ & $20-50$ & $600-1200$ & $1,3-4,4$ & $0,1-3,2$ & $240-1520$ & $6-7,9$ \\
\hline Espécies/Variáveis ambientais & $\begin{array}{l}\text { Sílica } \\
(\mathrm{mg} \mathrm{L})\end{array}$ & $\begin{array}{l}\text { Temp.água } \\
\left({ }^{\circ} \mathrm{C}\right)\end{array}$ & $\begin{array}{c}\text { Disco de } \\
\text { Secchi }(\mathrm{cm})\end{array}$ & $\begin{array}{l}\text { Condutividade } \\
\qquad(\mu \mathrm{S} \mathrm{cm})\end{array}$ & $\mathrm{pH}$ & $\begin{array}{c}\text { Coliformes } \\
\text { fecais }\end{array}$ & \\
\hline T. abrupta var. obesa & $\mathrm{NM}$ & 28 & NM & NM & $\mathrm{NM}$ & NM & \\
\hline T. conica & 10,8 & $27,4-30$ & 55 & 112,1 & 6,6 & NM & \\
\hline T. cylindraceae f. cylindraceae & NM & 27 & NM & NM & NM & NM & \\
\hline T. duquei & $8,14-26,8$ & $17,3-30,3$ & $25-45$ & $56-181,3$ & $6,0-7,0$ & $20-20000$ & \\
\hline T. helvetica & NM & 30 & NM & NM & NM & NM & \\
\hline T. megalacantha var. crenulatocollis & $8,1-8,4$ & $23,1-30,3$ & $25-30$ & $55,7-160,3$ & 6,8 & $170-20000$ & \\
\hline T. splendissima & $11,0-14,0$ & $12,8-13,1$ & 20 & 76,8 & 5,9 & 800 & \\
\hline T. verrucosa var. granulosa & NM & $26,9-28,7$ & 30 & $70,5-106,7$ & $7,4-7,6$ & NM & \\
\hline T. zingeri & $4,9-15,2$ & $12,8-19,7$ & $15-45$ & $71,7-132$ & $6,0-6,9$ & $800-2800$ & \\
\hline
\end{tabular}

Deflandre (1926) descreve a espécie como possuindo 10 cloroplastos sem pirenóides, nos exemplares ora analisados foi verificada a presença de pirenóides internos. Os indivíduos mostraram-se ligeiramente menores quanto ao comprimento que os espécimes dados por este mesmo autor, que cita dimensões de 28-32 $\times 20-24 \mu \mathrm{m}$, no entanto concordaram com o comprimento encontrado por Tell \& Conforti (1986) em material argentino. A variedade foi considerada rara por ocorrer somente na Ilha do Cravo na primavera de 1993.

2. Trachelomonas conica Playf. Proc. Linn. Soc. p.17, pl. 2, fig. 8, 9. 1915.

Fig. 3- 4

Lórica obovada, 20,3-21,2 $\mu \mathrm{m}$ compr., 13-14 $\mu \mathrm{m}$ larg., $\mathrm{Rc} / \mathrm{l}=1,5$; pólo anterior levemente achatado; poro com espessamento anelar ca. $3 \mu \mathrm{m}$; pólo posterior levemente acuminado; parede lisa, castanho-clara; cloroplastos numerosos, discóides ca. 2,5 ㅆm diâm.; pirenóide ausente.

Material examinado: BRASIL. Rio Grande do Sul: Ilha do Siqueira, 15/XII/1993, Costa (HAS26333); Foz do Rio Caí, 15/IX/1998, Torgan (HAS26825).
Distribuição geográfica: América do Sul, Ásia e Oceania. Primeira citação de ocorrência para o Rio Grande do Sul.

Comentários: a espécie foi considerada rara por ocorrer em apenas dois locais: na ilha do Siqueira na primavera de 1993 e na foz do rio Caí, no inverno de 1998.

3. Trachelomonas cylindraceae f. cylindraceae (Playf.) Pop. Opred Presnov. Vodor, 7: 97, pl. 27, fig. 5-7. 1955.

\section{Fig. 5-6}

Lórica elíptica a oblonga, 28-30 $\mu \mathrm{m}$ compr., 12-13 $\mu \mathrm{m}$ larg.; $\mathrm{Rc} / \mathrm{l}=2,3$; pólos arredondados, com colarinho cilíndrico, reto, com um espessamento anelar basal, ca. $3 \mu \mathrm{m}$ compr. por 4,5 $\mu \mathrm{m}$ larg.; parede lisa, castanho-avermelhada; cloroplastos numerosos ca. 3 um diâm.; pirenóides internos presentes; flagelo ca. 0,5 vezes o comprimento celular.

Material examinado: BRASIL. Rio Grande do Sul: Foz Sanga das Pedras, 15/XII/1993, Costa (HAS26332).

Distribuição geográfica: Ásia, Europa e Oceania. Primeira citação de ocorrência para as Américas, Brasil e Rio Grande do Sul. 
Comentários: Trachelomonas volzii Lemm. var. cylindraceae é sinonímia de $T$. cylindraceae $\mathrm{f}$. cyllindraceae, variedade que foi elevada à categoria de espécie por Popova (1955). Espécie considerada rara por só ocorrer na foz da Sanga das Pedras em amostra de espremido de macrófitas aquáticas, na primavera de 1993.

4. Trachelomonas duquei Conf. \& Nudelman. Rev. Hydrobiol. Tropical, 27(4): 304. pl. III, fig. 1-2. 1994.

Fig. 7

Lórica oblonga, 35-36,5 $\mu \mathrm{m}$ compr., 32-33 $\mu \mathrm{m}$ larg., $\mathrm{Rc} / 1=$ ca. 1,1 ; pólos arredondados a levemente achatados; parede pontuada, castanho-clara a castanho-avermelhada, com espinhos cônicos de até 2,8 $\mu \mathrm{m}$ compr., distribuídos irregularmente e espaçados; cloroplastídeos discóides, numerosos, ca. 2,8 $\mu$ m diâm.; pirenóides internos presentes.

Material examinado: BRASIL. Rio Grande do Sul: Foz Sanga das Pedras, 15/XII/1993, Costa (HAS26332); Ilha do Siqueira, 15/XII/1993, Costa (HAS26333); Ilha do Serafim, 7/V/1999, Alves-daSilva (HAS34635); Saco do Jacaré, 15/IX/1998, Alvesda-Silva (HAS34801); Saco do Cabral, 5/IV/1999, Alves-da-Silva (HAS34881); Saco dos Cachorros, 27/XII/1999, Salomoni (HAS34992); Ilha do Siqueira, 27/XII/1999, Alves-da-Silva (HAS34994).

Distribuição geográfica: América do Sul. Esta espécie foi descrita na Amazônia colombiana, sendo esta a primeira citação para o Brasil.

Comentários: esta espécie é muito semelhante a Trachelomonas allia var. obesa de Balech (1944: 30-32×23-25 $\mu \mathrm{m}$ ) diferindo, entretanto, por possuir maiores dimensões, pelos pólos levemente achatados e pelas pontuações bem marcadas na parede da lórica. A presença de pirenóides internos foi registrada pela primeira vez para esta espécie. Dentre as nove novas ocorrências foi o táxon mais amplamente distribuído ocorrendo em 39\% das amostras (Tab. 1).

5. Trachelomonas helvetica Lemm. emend. Defl. Kryptfl. Mark. Bradenburg, 3: 529. 1910.

Fig. 8-10

Lórica cilíndrico-cônicas, 38-40 $\mu \mathrm{m}$ compr.,

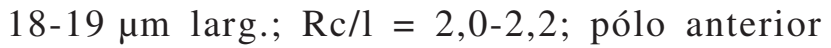
arredondado, lados regularmente arqueados; poro ca. $5 \mu \mathrm{m}$ diâm, formado pela fusão de espinhos cônicos ligeiramente truncados, 3-4 $\mu \mathrm{m}$ compr.; pólo posterior estreita-se abruptamente, terminando em processo caudal, curto, truncado; parede com espinhos curtos e cônicos, ca. $2 \mu \mathrm{m}$ compr., distribuídos em toda superfície da lórica; castanho-avermelhada; cloroplastos numerosos, discóides, ca. 3 um diâm.; pirenóide ausente.

Material examinado: BRASIL. Rio Grande do Sul: Ilha do Siqueira, 15/XII/1993, Costa (HAS26333).

Distribuição geográfica: América do Norte, Ásia, Europa e Oceania. Primeira citação da espécie para Brasil e Rio Grande do Sul.

Comentários: espécie considerada rara só sendo encontrada na ilha do Siqueira na primavera de 1993, com um a dois indivíduos por lâmina.

6.Trachelomonas megalacantha Cunha var. crenulatocollis Bourr. \& Manguin. Soc. d'Edition d'Enseignement sup. p. 184, pl. 23, fig. 322-323. 1952.

Fig. 13

Lórica elíptico-alongada, 64-65 $\mu \mathrm{m}$ compr., 45,5-46,2 $\mu \mathrm{m}$ larg.; Rc/l = ca. 1,4; pólos arredondados; poro flagelar ca. $6,5 \mu \mathrm{m}$ diâm.; espessamento anelar presente; colarinho formado pela fusão de espinhos cônicos, ca. $4 \mu \mathrm{m}$ compr. por 6,5-9 $\mu \mathrm{m}$ larg.; parede pontuada, avermelhada-clara; espinhos cônicos, distribuição irregular, retos ou levemente curvos, até $11 \mu \mathrm{m}$ compr.; cloroplastos numerosos, discóides, ca. 2,5 بm diâm.; pirenóide ausente.

Material examinado: BRASIL. Rio Grande do Sul: Saco do Cabral, 5/IV/1999, Alves-da-Silva (HAS34881); Ilha do Serafim, 5/IV/1999, Alves-daSilva (HAS 34885); Ilha do Cravo, 27/XII/1999, Alvesda-Silva (HAS34998).

Distribuição geográfica: América Central e América do Sul. Primeira citação de ocorrência no Rio Grande do Sul.

Comentários: esta variedade diferencia-se da espécie tipo por apresentar colarinho com bordo distal denteado, formado por espinhos curtos. No Brasil já foi encontrada no Estado do Amazonas por Uherkovich \& Schmidt (1974), Conforti (1993), Bittencourt-Oliveira (1997), no Paraná por Cecy (1990). No Parque foi encontrada no Saco do Cabral, ilha do Serafim e ilha do Cravo na primavera de 1998 e outono de 1999.

7. Trachelomonas splendidissima Middelh. Hydrobiologia 1(1): 87. pl. I, fig. 11-12. 1948.

Fig. 11-12

Lórica elíptico-ovada, 34-35 $\mu \mathrm{m}$ compr., 20,3-21,5 m larg., Rc/l = 1,6-1,7; pólos arredondados, pólo anterior estreita-se rapidamente em colarinho cilíndrico reto ou levemente inclinado, com margem levemente crenulada, 2-3 $\mu \mathrm{m}$ compr. por 5-6 $\mu \mathrm{m}$ larg.; pólo posterior ligeiramente mais largo com um grânulo 
central; parede granulada, castanho-avermelhada; cloroplastos discóides e bastoniformes ca. 2,5 $\mu \mathrm{m}, 8-9$ pirenóides internos; flagelo duas vezes o comprimento da lórica.

Material examinado: BRASIL. Rio Grande do Sul: Saco das Garças, 14/VI/1999, Alves-da-Silva (HAS34947).

Distribuição geográfica: Europa. Primeira citação para a América, Brasil e Rio Grande do Sul.

Comentários: a espécie foi encontrada na Holanda por Middelhoek (1948). Este autor a descreveu como possuindo 10-12 cloroplastos discóides, grandes e parietais, que bem poderiam representar a presença de pirenóides internos presentemente registrados. Espécie considerada rara, só encontrada no Saco das Garças, no outono de 1999.

8. Trachelomonas verrucosa Stockes var. granulosa (Playf.) Conr. \& Van-Meel. Mém. Inst. R. Sc. Nat. Belg. 124: 31, pl. 6, fig. 24. 1952.

Fig. 14-15

Lórica esférica, 13,8-14,5 $\mu$ m diâm.; poro flagelar ca. $2 \mu \mathrm{m}$ diâm.; espessamento anelar presente; colarinho levemente saliente com $0,5 \mu \mathrm{m}$ diâm., borda crenulada; parede levemente rugosa, castanhoavermelhada, grânulos irregulares em toda superfície da lórica; cloroplastos discóides, ca. $2 \mu \mathrm{m}$ diâm.; pirenóides internos presentes.

Material examinado: BRASIL. Rio Grande do Sul: Saco do Jacaré, 13/IV/1993, Rosa (HAS27079); Canal das Balseiras, 19/I/1999, Salomoni (HAS34908); Saco Santa Cruz, 1/IX/1999, Salomoni (HAS34922).

Distribuição geográfica: América do Norte, América do Sul, Ásia, Europa e Oceania. Primeira citação para o Brasil e para o Estado do Rio Grande do Sul.

Comentários: difere da variedade típica pela presença de grânulos irregulares, distribuídos em toda superfície da lórica, e pelas menores dimensões.

9. Trachelomonas zingeri Roll. Russ. arkh. Protist. 4:149, pl. 5, fig. 24. 1925.

Fig. 16

Lórica elíptica, 43-44,5 $\mu \mathrm{m}$ compr., 27,4-28,5 $\mu \mathrm{m}$ larg., Rc/l =1,5-1,6; pólos arredondados; poro flagelar ca. 2,5 um diâm.; espessamento anelar presente; colarinho baixo; parede pontuada, castanhoavermelhada, com espinhos cônicos, agudos, ca. $5 \mu \mathrm{m}$ compr.; distribuição irregular, distantes uns dos outros; cloroplastos bastoniformes, 3,7-4,6 بm diâm.; pirenóides internos.
Material examinado: BRASIL. Rio Grande do Sul: Saco do Cabral, 15/XI/1998, Alves-da-Silva (HAS34791); Saco do Cabral, 14/VI/1999, Alves-daSilva (HAS34945), Saco do Cabral, 27/IX/1999, Alvesda-Silva (HAS34959).

Distribuição geográfica: América do Norte, América do Sul e Europa. Primeira citação para o Estado do Rio Grande do Sul e terceira para o Brasil.

Comentários: esta espécie foi encontrada anteriormente no Estado do Amazonas por Thomasson (1971) e Menezes et al.(1995). No Parque só foi encontrada no Saco do Cabral, no inverno de 1999 e primavera de 1998/1999, com dois a três indivíduos por lâmina. Os indivíduos encontrados apresentaram-se ligeiramente maiores que o citado para Argentina por Tell \& Conforti (1986) que dão dimensões de 38-42x 22-22,5 $\mu \mathrm{m}$. Os indivíduos encontrados por Thomasson (1971) apresentaram dimensões de 46-48×30 $\mu \mathrm{m}$. Enquanto Menezes et al. (1995) encontrou espécimes com menores dimensões, 30-32×13-15 $\mu \mathrm{m}$. Portanto os espécimens de $T$. zingeri encontradas no Delta apresentaram dimensões intermediárias entre as medidas fornecidas por Tell \& Conforti (1986) e Thomasson (1971), e maiores que as encontradas por Menezes et al. (1995).

Somente 11 locais entre os 26 amostrados apresentaram novos registros de Trachelomonas: o saco do Cabral, Saco das Garças, Saco do Jacaré, Saco dos Cachorros, Saco Santa Cruz, ilha do Siqueira, ilha do Serafim, ilha do Cravo, foz Sanga das Pedras, foz do rio Caí e canal das Balseiras. Distinguindo-se a ilha do Siqueira e o Saco do Cabral com três táxons, respectivamente.

As espécies estudadas foram encontradas em águas com grande amplitude de variação de fatores ambientais como: a amônia variando de 12-600 $\mu \mathrm{g} \mathrm{L}$, nitrito de <1,3-131 $\mu \mathrm{g} \mathrm{L}$, nitrato de 500-2100 $\mu \mathrm{g} \mathrm{L}$, oxigênio dissolvido de 1,3-6,9 mg L, demanda bioquímica de oxigênio de 0,1-3,2 mg L, ortofosfato de 90-1520 $\mu \mathrm{g} \mathrm{L}$, matéria orgânica de 3,3-7,9 mg L, sílica de 4,9-25,8 mg L, disco de Secchi, 15-45 cm, condutividade $55,7-181,3 \mu \mathrm{S} \mathrm{cm}$, temperatura da água de $12,8-30{ }^{\circ} \mathrm{C}$, disco de Secchi de $15-55 \mathrm{~cm}$, coliformes fecais de 20-20.000 (em $100 \mathrm{~mL}$ ) e pH de 5,9-7,6 (Tab. 2).

Oito táxons podem ser considerados raros por serem encontrados no máximo em três locais dentre o total de amostras com novas citações, com exceção de Trachelomonas duquei, espécie que apresentou maior distribuição e maior tolerância as variáveis ambientais medidas (Tab. 1-2). 

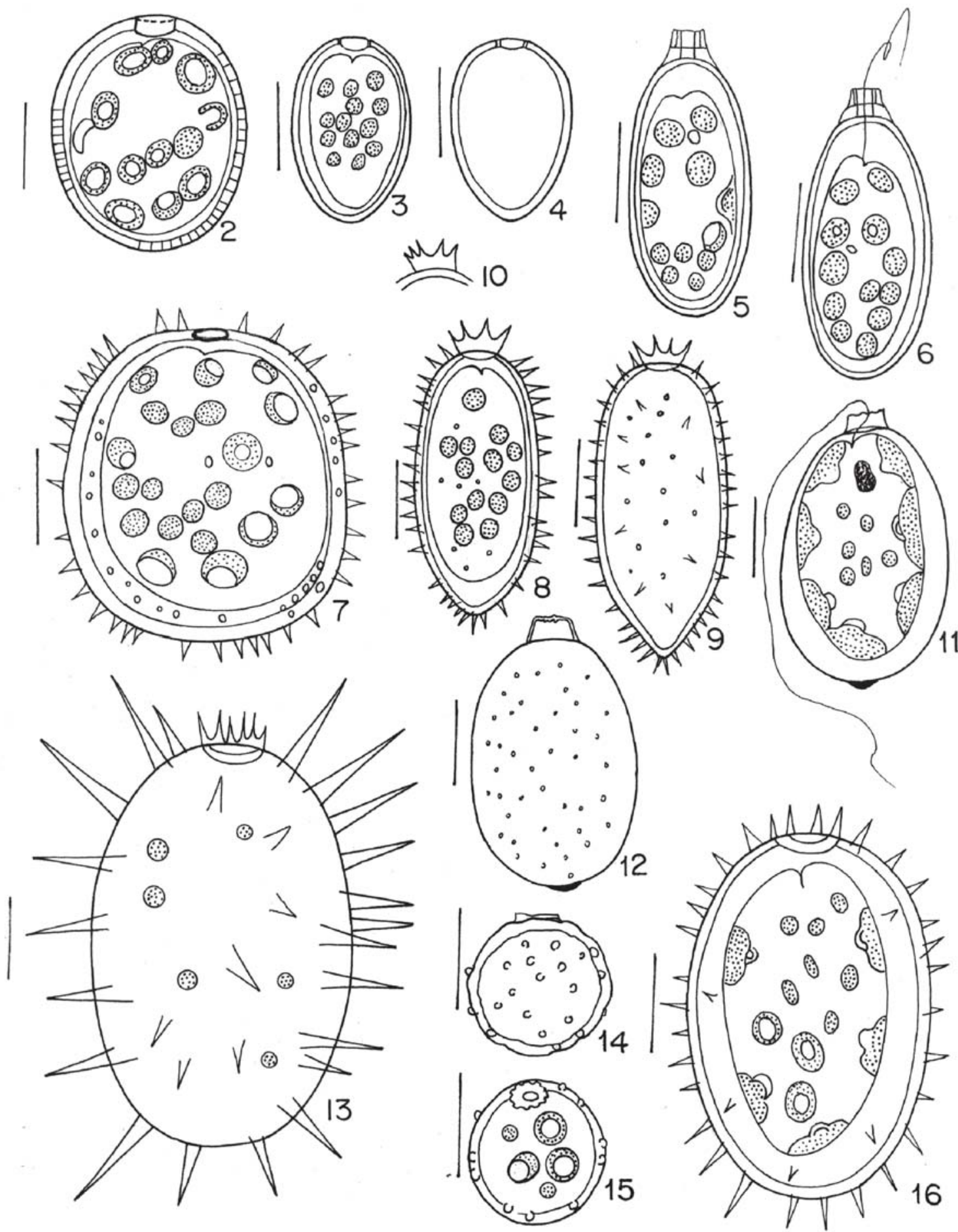

Figura 2-16. 2. Trachelomonas abrupta Swir. emend. Defl. var. obesa (Playf.) Defl. 3-4. T. conica Playf. 5-6. T. cylindraceae f. cylindraceae (Playf.) Pop. 7. T. duquei Conf. \& Nudelman. 8-10. T. helvetica Lemm. emend. Defl. 10. Detalhe do colarinho. 11-12. T. splendidissima Middelh. 13. T. megalacantha Cunha var. crenulatocollis Bourr. \& Manguin. 14-15. T. verrucosa Stockes var. granulosa (Playf.) Conr. \& Can-Meel. 16. T. zingeri Roll. (Escala = $10 \mu \mathrm{m})$. 
Oito novas citações de ocorrência do gênero Trachelomonas no Parque Estadual Delta do Jacuí foram encontrados no máximo em três continentes, enquanto Trachelomonas helvetica possui ampla distribuição sendo encontrada em quatro continentes.

Dois táxons são novos registros para a América: Trachelomonas cylindraceae f. cylindraceae, previamente só encontrada na Ásia, Europa e Oceania e Trachelomonas splendidissima registrada somente na Europa.

Um táxon é restrito às América Central e do Sul: Trachelomonas megalacantha var. crenulatocollis.

Três táxons são novos para o Brasil: Trachelomonas cylindraceae f. cylindraceae, T. helvetica e T. splendidissima.

Seis táxons são novos registros no Parque Estadual Delta do Jacuí e no Rio Grande do Sul: Trachelomonas abrupta var. obesa, T. conica, T. duquei, T. megalacantha var. crenulatocollis, T. verrucosa var. granulosa e T. zingeri.

\section{Agradecimentos}

Aos colegas do Museu de Ciências Naturais da Fundação Zoobotânica do Rio Grande do Sul, especialmente aos da Seção de Botânica de Criptógamas, pelo auxílio nas coletas das amostras; à Fundação de Amparo à Pesquisa do Rio Grande do Sul (FAPERGS), pela bolsa de Iniciação Científica (Proc. $n^{\circ}$ 0213491); à Rejane Rosa, pela cobertura à nanquim dos desenhos; aos colegas do laboratório de Geoprocessamento da Fundação Zoobotânica do Rio Grande do Sul, pela confecção do mapa.

\section{Referências bibliográficas}

Alves-da-Silva, S.M. \& Ávila, I.R. 1997. Levantamento florístico das Euglenaceae pigmentadas do Parque Estadual Delta do Jacuí, Rio Grande do Sul, Brasil. Iheringia 48: 85-102.

Alves-da-Silva, S.M. \& Bridi, F.C. 2004a. Euglenophyta no Parque Estadual Delta do Jacuí, Rio Grande do Sul, Sul do Brasil. 3. Strombomonas Defl. Acta Botanica Brasilica 18(3): 555-572.

Alves-da-Silva, S.M. \& Bridi, F.C. 2004b. Estudo de Euglenophyta no Parque Estadual Delta do Jacuí, Rio Grande do Sul, Brasil. 2. Os gêneros Phacus Dujardin e Hyalophacus (Pringheim) Pochmann. Iheringia, sér. Bot. 59(1):75-96.

Alves-da-Silva, S.M. \& Crossetti, L.O. 1999. Novas citações de ocorrência de Euglenaceae pigmentadas para o estado do Rio Grande do Sul, Brasil. Hoehnea 26(1): 47-60.
Alves-da-Silva, S.M. \& Hahn, A. 2001. Lista de Euglenophyta registradas em ambientes de águas continentais e costeiras do Estado do Rio Grande do Sul, Brasil. Iheringia 55: 171-188.

Alves-da-Silva, S.M. \& Hahn, A. 2004. Study of Euglenophyta in the Jacuí Delta State Park, Rio Grande do Sul, Brazil. 1. Euglena Ehr., Lepocinclis Perty. Acta Botanica Brasilica 18(1): 123-140.

American Public Health Association. APHA 1992. Standart methods for the examination of water and wastwater. 18 ed., Washington.

Balech, E. 1944. Trachelomonas de la Argentina. Anales del Museo Argentino de Ciencias naturales Bernardino Rivadavia 41: 223-305.

Bittencourt-Oliveira, M.C. 1997. Euglenophyceae do reservatório de Balbina, Estado do Amazonas, Brazil. Hoehnea 24(1): 21-40

Cecy, I.I.T. 1990. A Restinga do Pontal do Sul, município de Paranaguá, PR. 1. Levantamento ficológico (Euglenophyta) e físico-químico. Arquivos de biologia e tecnologia 33(1): 1-79.

Conforti, V.T.D. 1993. Study of the Euglenophyta from Camaleão lake (Manaus-Brazil). I. Trachelomonas. Revue Hydrobiologie tropicale 26(1): 3-18.

Conforti, V.T.D. \& Ruiz, L. 2001. Euglenophytes from Chuman Reservoir (South Korea) II Trachelomonas Ehr. Algological Studies 102: 117-145.

Conrad, W. \& Van-Meel, L. 1952. Materiaux pour une monographie de Trachelomonas Ehrenberg. C. (1834): Strombomonas Deflandre. G. (1930): et Euglena Ehrenberg C. (1832): genres d'Euglénacées. Mémoires d'Institute Royal des science naturelle Belgique 124: 1-176.

Deflandre, G. 1926. Monographie du genre Trachelomonas Ehrenberg. Nemours, André Lesot (cópia xerografada).

Huber-Pestalozzi, G. 1955. Euglenaceen. In: Das phytoplankton des Susswässers: Systematik und Biologie. Stuttgart: E. Schweizerbart'sche Verlangsbuchhandlug. v.16, n. 4.

Menezes, M.; Fonseca, C.G. \& Nascimento, E.P. 1995. Algas de três ambientes de águas claras do município de Parintins, estado do Amazonas, Brasil: Euglenophyceae e Dinophyceae. Hoehnea 22(1/2): 1-15.

Middelhoek, A. 1948. À propos de quelques espèces du genre Trachelomonas Ehr. et du genre Strombomonas Defl. trouvées aux Pays-Bas. I. Hydrobiologia 1(1): 78-79.

Németh, J. 1980. Az ostoros Algák (Euglenophyta). Budapeste, Vizdock. (Hidrobiologia Series n. 8).

Popova, T.G. 1955. Euglenovyge vodorosli. Opredenlitel presnovodnynch SSSR. Sov. Nauka 7: 1-267.

Shi, Z.; Wang, Q.; Xie, S. \& Daí, J. 1999. Euglenophyta. Science Press. 414p., il. (Flora algarum sinicarum aquae dulcis, T. 6)

Starmach, K. 1983. Euglenophyta. In: K. Starmach. Flora Slodkowodna Polski. 3.Warszawa, Polska Academia Nauk.

Tell, G. \& Conforti, V.T.D. 1986. Euglenophyta pigmentadas de la Argentina. Stuttgart, J. Cramer. Bibliotheca Phicologica 75: 163-182.

Thomasson, K. 1971. Amozonian algae. Institut Royal des Sciences Naturelles de Belgique. $2^{\circ}$ sér., 86. 
Uherkovich, G. \& Schmidt, A. 1974. Phytoplankton taxa in dem zentralamazonischen Schwemmlandsee Lago do Castanho. Amazoniana 5(2): 243-283.
Wolowski, K. 1998. Taxonomic and environmental studies on euglenophytes of the Kraków-Czestochowa Upland (Southern Poland). Fragmenta Floristica et Geobotanica v. 6, p. 1-192. Suplements. 